\title{
Short communication: Measuring feed volume and weight by machine vision
}

\author{
A. N. Shelley, ${ }^{*}$ D. L. Lau, ${ }^{*}$ A. E. Stone, $\dagger$ and J. M. Bewley $\dagger^{1}$ \\ *Department of Electrical Engineering, and \\ †Department of Animal and Food Sciences, University of Kentucky, Lexington 40546
}

\begin{abstract}
Individual dairy cow feed intake is closely related to the health and productive output of each cow, with healthy cows generally eating more feed than unhealthy cows. Incorporating the use of an automated system to monitor feed consumption for each cow may be beneficial for dairy farm management. This study examined the use of an inexpensive 3-dimensional video camera to measure feed volume, from which we derived feed weight. Proof-of-concept testing was conducted to determine the effectiveness and capability of the machine vision feed-scanning system and its possible use in feed intake monitoring. Such systems are ideal because they do not impede the workflow of the farm or interrupt feeding behavior. This is an improvement over existing systems that are labor and cost intensive. Our conducted experiments involve measuring feed volume at known weights, up to $22.68 \mathrm{~kg}$, with the resulting volume and weight values analyzed by means of linear and quadratic least squares $t$-test regression analysis. The effects of feed positioning in the bin and near-range sensor limitations were also examined. The results showed that an estimation of feed weight from 3-dimensional scan of volume measurements could be made to within $0.5 \mathrm{~kg}$ of the physically measured feed weight using a digital scale. Future efforts will focus on extending this work to active bunks with multiple cows eating throughout the day and testing total mixed rations of varied composition.
\end{abstract}

Key words: machine vision, structured light illumination, feed intake, precision dairy farming

\section{Short Communication}

Precision dairy farming is the use of technologies to measure physiological, behavioral, and production indicators in individual animals to improve management strategies and farm performance (Bewley and Schutz, 2010). Monitoring dairy cow feed intake can simply

Received October 12, 2014.

Accepted September 7, 2015.

${ }^{1}$ Corresponding author: jbewley@uky.edu utilize human visual inspection, such as in the research conducted by Bach et al. (2004), but the difficulty in manually collecting data at the time of feeding limits the extent of this type of monitoring (Friggens et al., 1998). Several nutrition models have been developed to attempt to predict feed intake, but even the best models have only been able to account for up to $70 \%$ of the variation in intake (VandeHaar, 1998) and all of the models underpredict actual observed DMI (Rim et al., 2008).

An automated feed monitoring system has the potential to monitor individual cow feed intake electronically. Radio frequency identification (RFID) is the typical means used to monitor individual cows. Examples of RFID-based systems include the GrowSafe system (GrowSafe Systems Ltd., Airdrie, AB, Canada) and Calan gates (American Calan Inc., Northwood, NH). Schwartzkopf-Genswein et al. (1999) and DeVries et al. (2003) monitored the feeding patterns of feedlot cattle with an RFID system. They concluded that the RFID nature of the system had some inherent factors that produced errors, meaning that actual feeding data had the potential to go unrecorded (Schwartzkopf-Genswein et al., 1999; DeVries et al., 2003).

The Insentec (Insentec, Marknesse, the Netherlands) monitoring system permits loose-housed cows to freely access several feeding and drinking stations, which allows researchers to collect continuous feeding and drinking behavioral data. The basis of this system is RFID coupled with an automated barrier between the cow and the feed and water. The Insentec monitoring system performed well in the study by Chapinal et al. (2007), but it still interfered with the natural feeding behaviors of the cow as a physical barrier was placed between the cow and the feed. The ideal system measures, controls, and monitors individual feed intake of the free-housed cow while not interfering with feeding habits and not introducing additional work or inhibiting workflow on the farm (Halachmi et al., 1998).

The basis of this research relied upon the ability to determine the volumetric mass density of a TMR simply by determining how much mass of the TMR can be contained within a known volumetric container. Because TMR are not solid but instead contain air 
pockets due to the particulate nature of the TMR, bulk density is the preferred volumetric basis. The physical properties and moisture content of a TMR change negligibly over a 24 -h period; therefore, the volumetric shape of the TMR can be used to determine the weight of the feed directly. By knowing any 2 of the 3 variables (density, volume, and mass), the third can be directly calculated.

The objective of this study was to assess the proofof-concept usefulness of an inexpensive 3-dimensional (3D) video camera for measuring individual dairy cow feed intake by scanning and recording feed volume, from which we indirectly derived feed weight. The overall procedure was to record 3D scans of a feed bin at various fill levels and, from the resulting scale weight versus feed volume, derive a single mapping from volume to weight. The resultant mapping was then used to compare the weight of feed derived from the volume to the actual weight measured by a digital scale. The differences between these 2 values were analyzed by regression analysis. Simple regression analysis was conducted on all experimental setups tested. We chose regression analysis because the objective was to determine if a strong correlation existed between the camera sensor depth measurements of the feed surface in a bin and the scale-measured weight values of feed in a bin. A linear correlation was assumed but a quadratic correlation was utilized to expose inherent sensor error when the camera was positioned from the feed at or below the lower optimal operating range. Sensor noise at close range has been previously studied and can be modeled as a quadratic relationship, as outlined in the work of Nguyen et al. (2012).

To establish a data set of weights and volumes, the same plastic feed bin used in controlled research feeding at the University of Kentucky Coldstream Dairy Research Farm was used in this research. The bin was a Rubbermaid 3501 Food/Tote Box (Rubbermaid Commercial Products LLC., Winchester, VA) with the following dimensions: $66.0 \mathrm{~cm}$ long, $45.7 \mathrm{~cm}$ wide, and $38.1 \mathrm{~cm}$ deep for a total volume capacity of $81.4 \mathrm{~L}$ or $0.29 \mathrm{~m}^{3}$ and a maximum weight capacity of $23.98 \mathrm{~kg}$. A digital scale, Royal 17016G 315-lb freight scale (Royal Consumer Information Products Inc., Bridgewater, NJ), which measures weight with an accuracy of $\pm 0.0045 \mathrm{~kg}$, was used to obtain feed weight measurements.

For measuring volume, we used a PrimeSense Carmine 1.08 RGB+depth sensor (PrimeSense, Tel Aviv, Israel), which works by projecting a pseudorandom dot pattern from a near-infrared laser illuminator and then using a near-infrared camera to detect constellations of points. The position of these constellations within the camera's field of view determines how far the target surface is from the sensor. Positioning the sensor over the feed bin then creates an opportunity to measure the feed in the bin simulating the times before and after a cow has fed from the bin.

As with any machine vision system, no camera can make measurements occluded from its view, such as that of feed hidden at the bottom of a bin when imaged from the side. For this reason, measuring feed volume must be done either by using a scanning process of sweeping the camera over the feed to ensure that no portion of the feed is occluded from the camera, such as in the first and second experimental setups, or by placing the camera directly above the feed bin to ensure no portion of the bin occludes the feed, such as in the third experimental setup. KScan3D software (LMI Technologies Inc., Delta, BC, Canada) was used to capture images and to merge images in real time.

The sensor was installed onto an 80/20 aluminum frame (80/20 Inc., Columbia City, IN) to minimize the effects of camera motion on the merging process. A pivot arm was constructed to bring the camera over the center of the feed bin for scanning or to move the camera out of the scan area. Attached to the side of the pivot arm was a swivel arm that allowed for $360^{\circ}$ of free rotation beneath the pivot arm about the point of attachment. The camera was manually positioned in the same location for the start of every scan in a test set. In the first experimental setup, the camera was at a distance of $99.1 \mathrm{~cm}$ from the floor and $61.0 \mathrm{~cm}$ from the top of the feed bin. The camera was intentionally placed at a distance from the feed surface below the 80-cm lower limit of the 3D depth sensor's optimal operating range to observe the effects on the accuracy of the image data.

For the merged image tests of the first and second experimental setups, the first image functioned as the anchor to which all of the successive images would be automatically merged and set the Cartesian coordinates to be assigned to all 3D scan data. The software then proceeded to collect 13 more images with a 2 -s delay between each image capture as the user manually rotated the camera clockwise (looking down onto the bin) through one revolution with the swivel arm. As each image was captured, the KScan3D software automatically aligned and merged the new data with the previously merged data to produce an updated 3D surface.

A 0-kg, or empty bin, scan was the first scan collected with the system. The bin was then filled with $2.27 \mathrm{~kg}$ of feed, and the feed biased and scanned in 7 different positions within the bin: feed with a flat top surface; feed biased in the back left corner; back right corner; front left corner; front right corner; biased in the center of the bin; and feed with a hole in the center or the feed pushed out toward the walls of the bin. Biasing 
with respect to this research meant that the majority of the feed volume in the bin was concentrated in that area of the bin. The biasing of feed was conducted to determine if it had any effects on the system, as a robust system for measuring the feed volume and weight should not be affected by the surface contour of the feed. After collecting data on the $2.27-\mathrm{kg}$ bin of feed, the bin was then filled with another $2.27 \mathrm{~kg}$ of feed, and the same 7 biasing scans collected by the system. This process was then repeated at $2.27-\mathrm{kg}$ increments until the bin had $22.68 \mathrm{~kg}$ of feed, at which point the plastic bin could not hold any additional feed without spilling over the edges or being heavily mounded in the center of the bin. The DM content of the TMR tested in each experimental setup is shown in Table 1 .

After all scans had been conducted, the KScan3D software was then used to reduce the $3 \mathrm{D}$ point cloud data to $10 \%$, or roughly 80,000 data points, of the original data, as this was all that was needed to accurately represent the original $3 \mathrm{D}$ scan. The data were then processed to (1) align the bins with the origin and the Z-equals-zero plane; (2) interpolate the scattered data of each scan; (3) calculate the difference of the current volume scan data file from the $0-\mathrm{kg}$ (empty bin) scan data file; (4) create a new scan data set to represent the feed volume by itself (without the bin); (5) determine the numerical cumulative depth average value corresponding to the averaged volume value of the feed surface; and (6) analyze the relationship of the $3 \mathrm{D}$ scanned volume values to the known scale-measured weight values by regression analysis.

Aligning the bins with the origin ensured that the corner with the lowest data value for both bins would lie as close to the origin as possible. Aligning the bins with respect to the Z-equals-zero plane gave the data a view normal to the surface of the feed and the bin, as if the observer was looking directly down onto the bin and feed. The alignment of the bins allowed for interpolation of each data set to remove duplicate data for the feed surfaces, ensuring an accurate representation of the actual surface. By taking the difference of the 2 data sets, the data that would have otherwise represented the plastic bin, and not the feed in the bin, could be removed from the final volume calculation.

In the second experimental setup, the camera was moved to a distance of $138.1 \mathrm{~cm}$ from the floor and $100.0 \mathrm{~cm}$ from the top of the feed bin. The reason for this was to move the camera to a distance where the feed surface would be within the camera's optimum operating range of 80.0 to $350.0 \mathrm{~cm}$. Outside of this range, intrinsic errors from the camera itself are induced in the depth measurement data. The same experimental procedures conducted in the first experimental setup were repeated for this increased camera distance setup.
Table 1. Ingredient and DM content of the TMR tested in the experimental setups ${ }^{1}$

\begin{tabular}{lcc}
\hline Composition (\% of DM) & TMR 1 & TMR 2 \\
\hline Corn silage & 44.6 & 34.3 \\
Alfalfa silage & 25.6 & 12.7 \\
Cottonseed whole & 2.8 & 5.8 \\
Alfalfa hay & 2.8 & 4.9 \\
Grain mix & 24.2 & 42.3 \\
\hline
\end{tabular}

${ }^{1}$ TMR 1 was tested in the first experimental setup; TMR 2 was tested in the second and third experimental setups.

No further camera distance changes were tested as any increased distance from sensor to scene would only reduce system accuracy.

In the third experimental setup, the camera was kept at $138.1 \mathrm{~cm}$ from the floor and $100.0 \mathrm{~cm}$ from the top of the feed bin, but a single image was captured from directly above the center of the feed surface. This test was conducted to determine if a single image capture system could derive comparable image weight values as the first 2 setups tested without requiring multiple image captures, a rotation mechanism about the feed surface, or the need for computationally intensive image merging. The same data processing was conducted on the data collected in the third experimental setup as outlined for the first and second experimental setups. This data processing included the same ordered operation steps of data reduction, alignment, interpolation, differencing, cumulative feed surface depth average value derivation, and simple regression analysis as outlined in the data processing description of the first experimental setup.

Figure 1 shows the first- and second-order least squares model fits for all 3 experimental setups. The results show a strong model fit $\left(\mathrm{R}^{2}>0.99\right)$ for both linear and quadratic modeling of the volumetric scan data with relation to scale-measured weight values. The initial hypothesis was that the relationship would be strictly linear. The residual error seen in Figures $2 \mathrm{a}$ and $2 \mathrm{~b}$ was determined to be mainly due to the inherent sensor noise error of the PrimeSense camera's depth sensor at short distances, as expected. In the first experimental setup tested, where the sensor was intentionally placed below the manufacturer-suggested lower operating range, the increasing variance directly due to the increasing sensor noise error is clearly visible. We determined that a second-order fit instead would be optimal, as it would include correction compensation for this residual sensor error at distances near this lower operating range. Figures $2 \mathrm{c}$ and $2 \mathrm{e}$ continue to exhibit a slight nonlinear relationship between the scale weight and the image volume, which was still due to sensor noise but to a lesser degree as these experimental setups tested bin weights within the optimal operating 
range of the camera sensor. At the increased camera distance in the second and third experimental setups, the contribution of sensor noise was greatly reduced and the variance randomly distributed when a quadrat- ic relationship between scale weight and image volume was considered, as can be seen in Figures $2 \mathrm{~d}$ and $2 \mathrm{f}$.

The linear standard error values achieved for the 3 experimental setups, in order, were $0.76,0.56$, and

a)

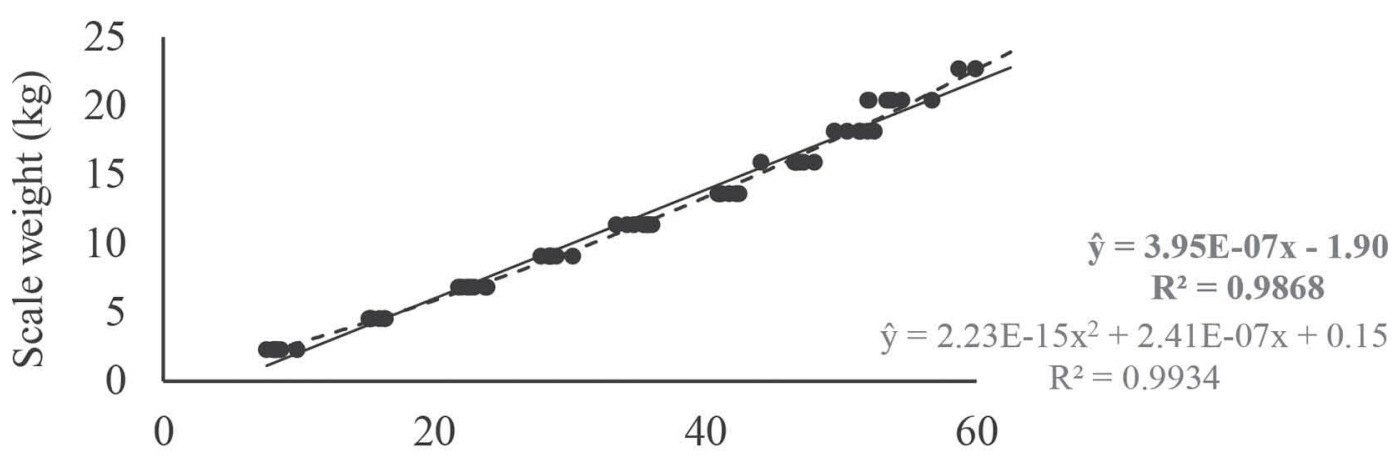

b)

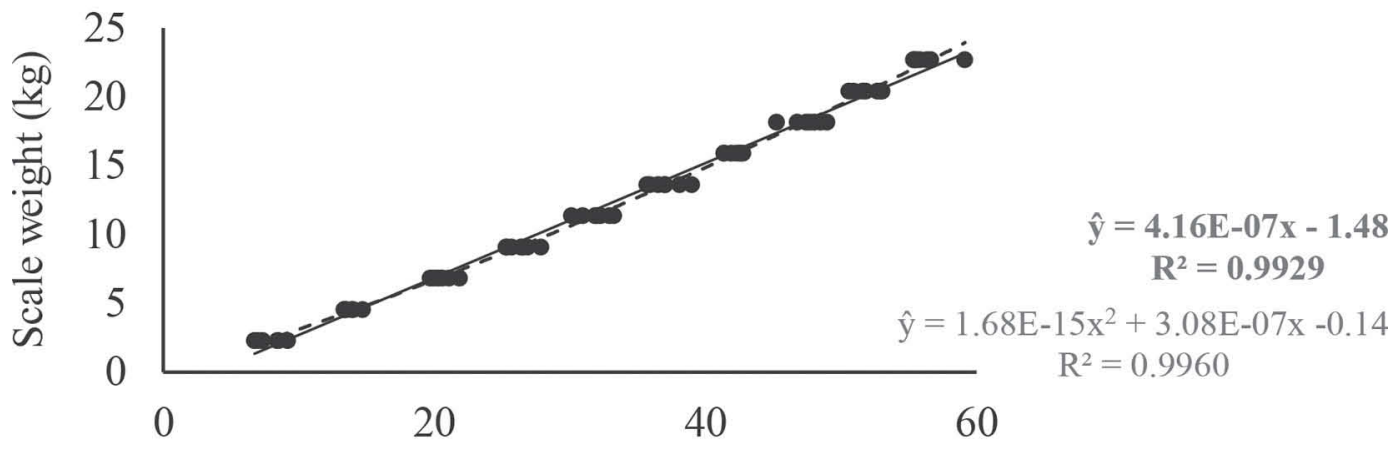

c)

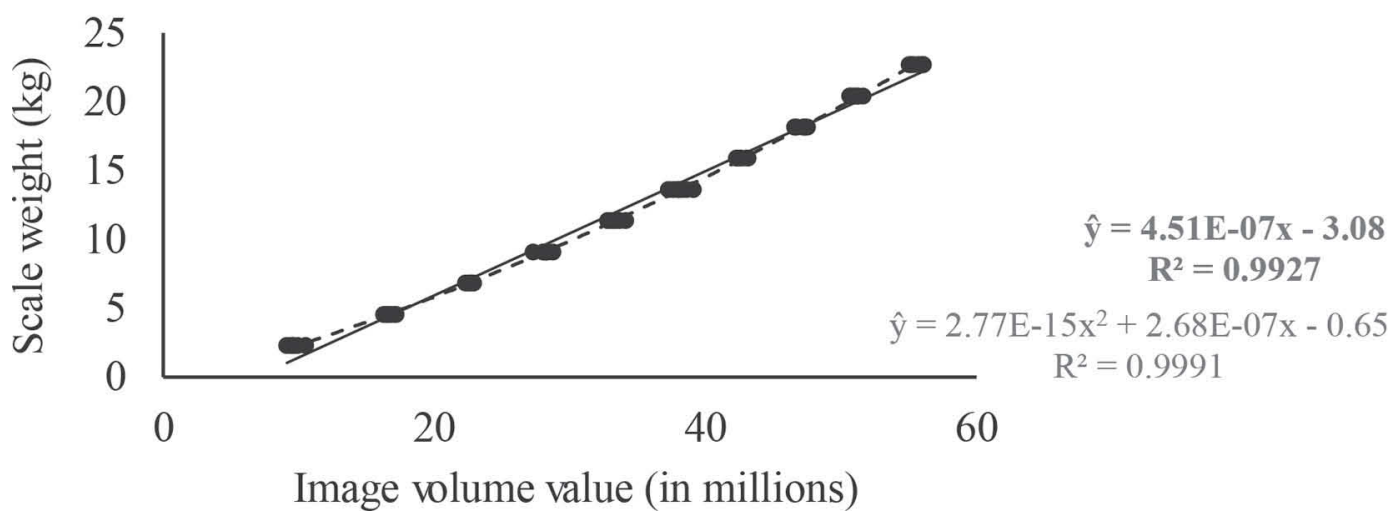

Figure 1. The collected data samples were plotted along with linear (solid line) and quadratic (dashed line) least squares regression models fit to the data comparing the image volume value to the scale weight for the first (a), second (b), and third (c) experimental setups tested. The equations of fit and $\mathrm{R}^{2}$ values are shown at right for linear models (in bold) and quadratic models (in gray). These results showed that although a strong linear relationship existed between the scale-measured weight and 3-dimensional volumetric scan data variables $\left(\mathrm{R}^{2}=0.99\right)$, a quadratic fit provided a slightly stronger relationship $\left(\mathrm{R}^{2}>0.99\right)$. 
a)

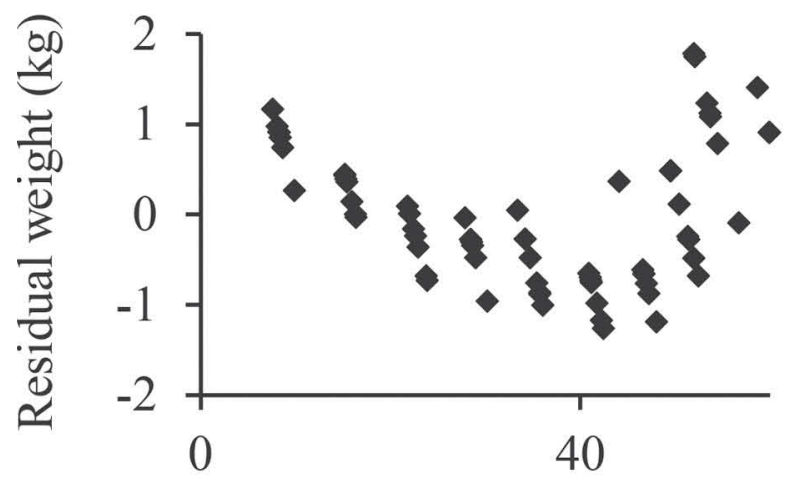

c)

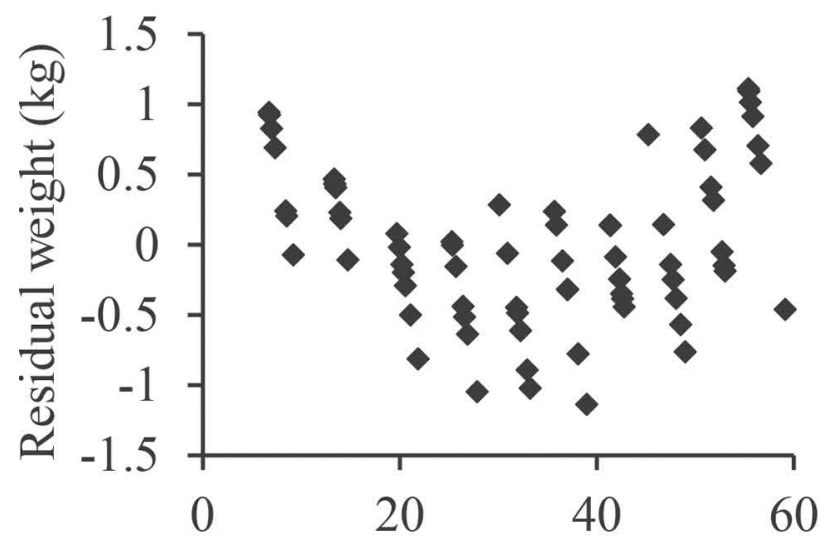

e)

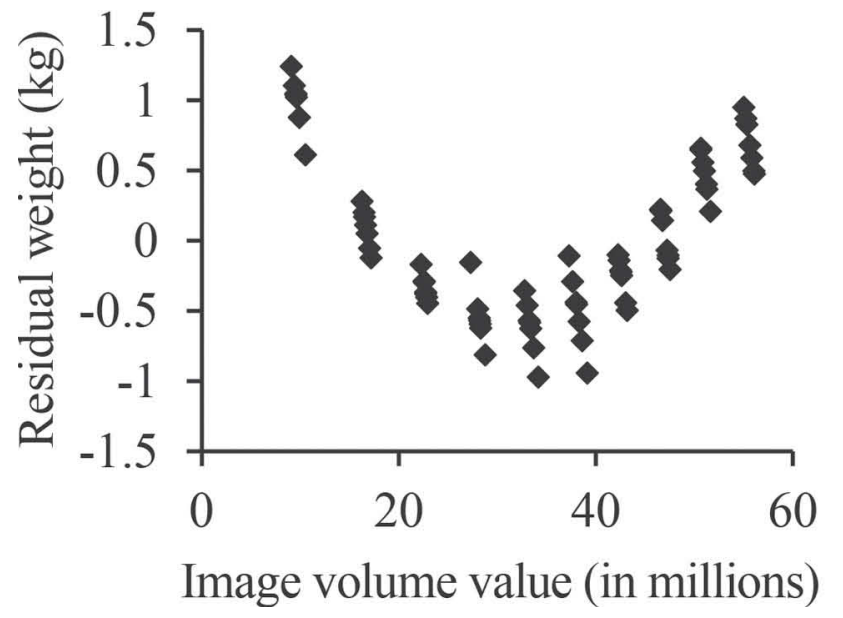

b)

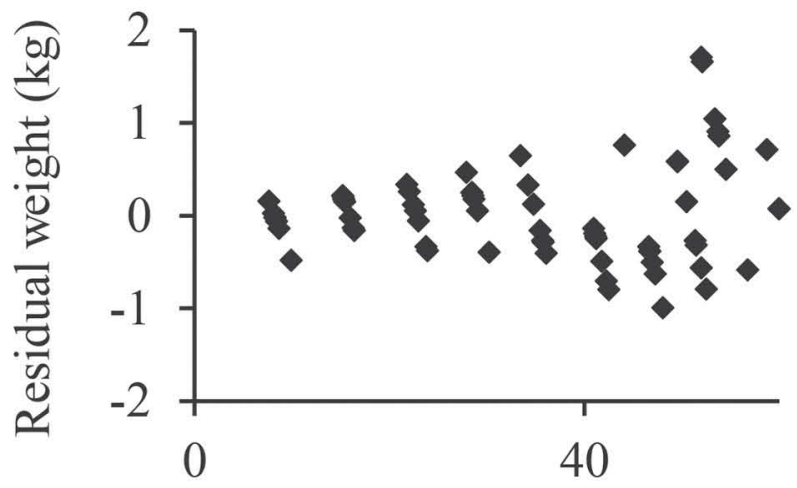

d)

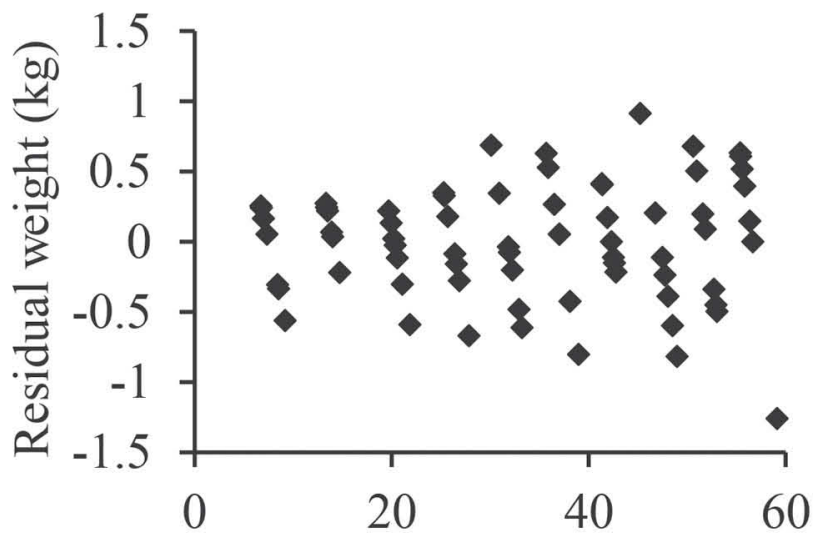

f)

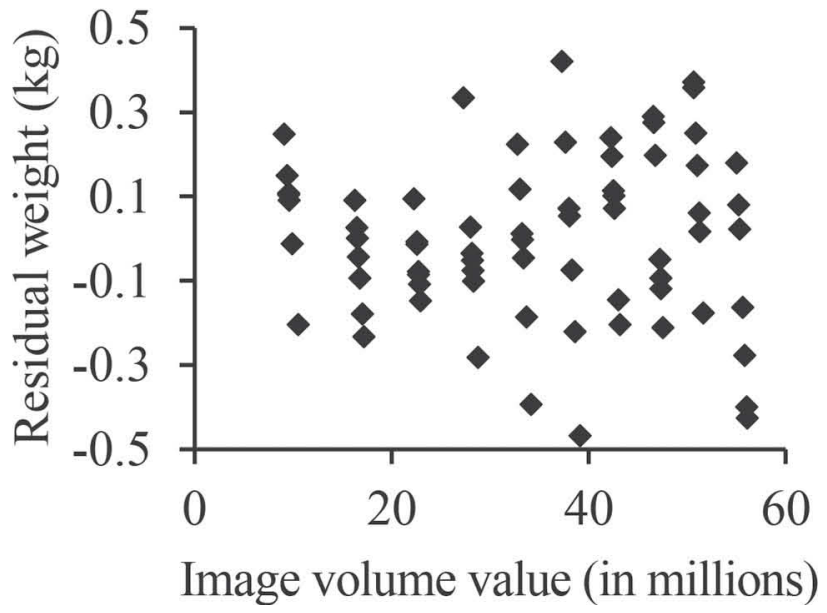

Figure 2. The linear residual error plots for the first (a), second (c), and third (e) experimental setups suggested that a nonlinear model may be a better fit than a linear model. The quadratic residual error plots for the first (b), second (d), and third (f) experimental setups suggested a nonlinear fit and a stronger quadratic relationship of image volume to scale weight. The inherent sensor noise error is the major component of this better quadratic model fit. 
$0.57 \mathrm{~kg}$. The single coefficients for the 3 linear models were all highly significant $(P<0.01)$. The quadratic standard error values achieved for the 3 experimental setups, in order, were $0.54,0.42$, and $0.20 \mathrm{~kg}$. The $P$ values for the individual coefficients of the 3 quadratic models were all highly significant $(P<0.01)$. These results show that the second-order model with a single image capture system had the least statistical error and, therefore, the best model fit to the data.

The fact that the residual errors for all 7 biased image captures for each weight increment in the quadratic residuals did not vary by more than $2 \mathrm{~kg}$ in the second experimental setup and by no more than $1 \mathrm{~kg}$ in the third experimental setup showed that variations in the surface, where the feed creates peaks and valleys in the surface, did not inhibit the system from accurately producing image weight values. As long as the system is calibrated before use at differing known scale weight values of the TMR, the system is able to produce image weight values to within a small percentage of error regardless of how the surface of the feed is arranged. Based upon the results of the 3 experimental setups, the single image capture system at a distance of 100.0 $\mathrm{cm}$ from the highest point of feed surface, which includes quadratic sensor error compensating conversion of volume to weight was deemed the best option in regard to both error minimization and practicality. A single image capture system can be run in real time for continuous monitoring of the feeding area with no obstruction to the workflow or the cow's feeding behavior. The single image capture setup also has the benefit of having no moving parts that could result in mechanical failure, as well as having the smallest operating space requirement.

This study demonstrates potential for the use of imaging to measure feed intake, feed volume, and feed weight. Future studies should address how the system functions in open feedbunks with multiple cows entering and exiting throughout the day, as well as studying how feeding behaviors affect system accuracy. Facial recognition techniques or RFID may be used to identify when animals enter or exit the feedbunk and are actively eating. Separation panels extending from headlocks may be necessary if issues occur with cows entering their neighbors' defined areas to steal feed.
Variations in TMR content and density would necessitate a change in calibration when making major ration changes or in evaluating rations across farms. This research was conducted in a laboratory setting. Future experiments should carefully monitor changes in TMR density that could result from sorting of the ration by cows, thus changing the forage-to-concentrate ratio and (or) moisture content in the feed bunk. Changes in ration density through time represent a potential technical challenge for this approach.

\section{ACKNOWLEDGMENTS}

The authors acknowledge financial support from the Kentucky Science and Engineering Foundation.

\section{REFERENCES}

Bach, A., C. Iglesias, and I. Busto. 2004. Technical note: A computerized system for monitoring feeding behavior and individual feed intake of dairy cattle. J. Dairy Sci. 87:4207-4209.

Bewley, J. M., and M. M. Schutz. 2010. Recent studies using a reticular bolus system for monitoring dairy cattle core body temperature. Pages 218-219 in Proc. First North American Conference on Precision Dairy Management, Toronto, Canada. Precision Dairy Management, Woodstock, ON, Canada.

Chapinal, N., D. M. Veira, D. M. Weary, and M. A. G. von Keyserlingk. 2007. Technical note: Validation of a system for monitoring individual feeding and drinking behavior and intake in grouphoused cattle. J. Dairy Sci. 90:5732-5736.

DeVries, T. J., M. A. G. von Keyserlingk, D. M. Weary, and K. A. Beauchemin. 2003. Technical note: Validation of a system for monitoring feeding behavior of dairy cows. J. Dairy Sci. 86:3571-3574.

Friggens, N. C., B. L. Nielsen, I. Kyriazakis, B. J. Tolkamp, and G. C. Emmans. 1998. Effects of feed composition and stage of lactation on the short-term feeding behavior of dairy cows. J. Dairy Sci. 81:3268-3277.

Halachmi, I., Y. Edan, E. Maltz, U. M. Peiper, U. Moallem, and I. Brukental. 1998. A real-time control system for individual dairy cow food intake. Comput. Electron. Agric. 20:131-144.

Nguyen, C. V., S. Izadi, and D. Lovell. 2012. Modeling kinect sensor noise for improved 3D reconstruction and tracking. Pages 524-530 in Proc. 2012 Second Int. Conf. 3D Imaging, Modeling, Processing, Visualization and Transmission (3DIMPVT). IEEE, New York, NY.

Rim, J., S. Lee, Y. Cho, E. Kim, J. Kim, and J. Ha. 2008. Prediction of dry matter intake in lactating holstein dairy cows offered high levels of concentrate. Asian-australas. J. Anim. Sci. 21:677-684.

Schwartzkopf-Genswein, K. S., C. Huisma, and T. A. McAllister. 1999. Validation of a radio frequency identification system for monitoring the feeding patterns of feedlot cattle. Livest. Sci. 60:27-31.

VandeHaar, M. J. 1998. Efficiency of nutrient use and relationship to profitability on dairy farms. J. Dairy Sci. 81:272-282. 